58510

Thin Films and Surface Patterning with BEDT-TTF Based Charge Transfer Salts*

\author{
H. H. Wang 1 , C. Y. Han 1 , D.-Y. Noh ${ }^{2}$, K.-S. Shin ${ }^{2}$, G. A. Willing1 ${ }^{1}$ and U. Geiser ${ }^{1}$
}

${ }_{1}^{1}$ Materials Science Division, Argonne National Laboratory, Argonne, Il 60439, USA

2Department of Chemistry, Seoul Women's University, Seoul, Korea

Submitted for publication in

Synthetic Metals

Proceedings of the International Conference on Synthetic Metals

Shanghai, China

June 29-July 5, 2002

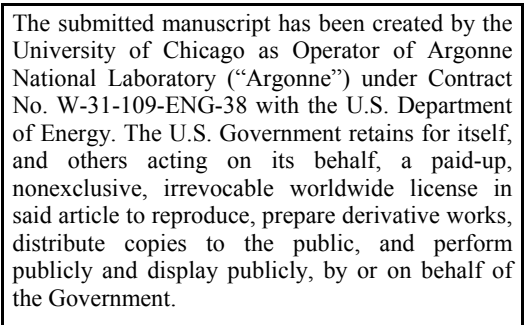

*Work supported by the U.S. Department of Energy, BES-Materials Sciences, under Contract W-31-109-ENG-38. 


\title{
Thin Films and Surface Patterning with BEDT-TTF Based Charge Transfer Salts
}

\author{
H. Hau Wang ${ }^{a}$, ${ }^{\text {Catherine Y. Han a }}$, Dong-Youn Noh ${ }^{\mathrm{b}}$, Kyong-Soon Shin ${ }^{\mathrm{b}}$, \\ Gerold A. Willing ${ }^{\mathrm{a}}$, Urs Geiser ${ }^{\mathrm{a}}$, \\ ${ }^{a}$ Materials Science Division, Argonne National Laboratory, Argonne, IL 60439, USA \\ 'Department of Chemistry, Seoul Women's University, Seoul, 139-774, KOREA
}

\begin{abstract}
Densely covered (ET) $\mathrm{X}_{2}$ thin films can be selectively electrodeposited on gold electrodes. The insulating $(\mathrm{ET}) \mathrm{X}_{2}$ films are converted to $(\mathrm{ET})_{2} \mathrm{X}$ conductive films through a novel conproportionation reaction. Both ET and ET salt patterns can be prepared with the PDMS stamping technique with use of an ET derivative with a dodecanethiol chain for surface derivatization. These solution procedures open up the possibility to prepare conductive and superconductive charge-transfer salt thin films as well as patterns.
\end{abstract}

Keywords: Electrocrystallization, BEDT-TTF, Self-assembly using surface chemistry, Polycrystalline thin films, Raman spectroscopy

\section{Introduction}

Organic conductors and superconductors based on charge transfer salts such as (BEDT-TTF $)_{2} \mathrm{X}$ have been studied extensively during the past twenty years, where BEDT-TTF or ET is bis(ethylenedithio)-tetrathiafulvalene. More than 360 ET salts have been reported including mono- and divalent anions, same anion but different stoichiometries $\left(\mathrm{ET}_{\mathrm{m}} \mathrm{X}_{\mathrm{n}}\right)$, same stoichiometry but different crystal packing motifs (such as $\alpha, \beta, \gamma$, phases etc.), and with or without co-crystallized solvent molecules.[1, 2] Many interesting physical phenomena have been observed such as solid-state to solid-state thermal transformation ( $\alpha$ to $\beta$ phase), pressure induced phase transformation ( $\beta$ to $\beta^{*}$ phase), charge density wave, metal-to-insulator, antiferromagnetic,[3] spin-Peierls,[4] ferromagnetic,[5] and superconductive phase transitions, etc. These temperature, pressure, and magnetic field induced phase transitions may lead to various sensor and electronic applications. In order to shape these charge transfer salts

\footnotetext{
${ }^{*}$ Corresponding author Tel: 630-252-3461; fax 630-252-9151; E-mail: hau.wang@anl.gov. Work at Argonne National Laboratory is sponsored by the U. S. Department of Energy, Office of Basic Energy Sciences, Division of Materials Sciences, under Contract W-31-109-ENG38. Work at Seoul Women's University is supported by the Korea Research Foundation Grant (KRF-2001-013-D0043).
}

for both fundamental studies and potential device application, the need for thin films and surface patterning is evident.

\section{Thin Film of $(E T)_{2} \mathrm{PF}_{6}$}

To date, reported efforts to prepare charge transfer organic thin films include chemical/physical vapor deposition, reticulate polymer matrix technique, and Langmuir-Blodgett approach. The first two methods work well only with volatile chemical species. It is our interest to develop a general solution based procedure because most of the known ET salts were prepared from solution based electrocrystallization with non-volatile reagents. Our procedure to prepare ET based thin films involves two steps and the details have been described recently.[6] The first step is to generate over-oxidized (ET) ${ }^{2+}$ salts with use of much higher current density $\left(>20 \mu \mathrm{A} / \mathrm{cm}^{2}\right)$ than that of the conventional electrocrystallization $\left(<5 \mu \mathrm{A} / \mathrm{cm}^{2}\right)$. The process generates high concentration of $(\mathrm{ET})^{2+}$ species that is insulating and leads to a passive film that covers the entire anode. This electrochemical process is selective. The $(\mathrm{ET})^{2+}$ species only cover the electrode surfaces but not the surrounding substrate. This nature leads to surface patterning capability. The second step involves a novel conproportionation reaction where $\mathrm{ET}(\mathrm{X})_{2}$ is chemically 
reduced by neutral ET molecules from solution according to the following overall equation:

$$
(\mathrm{ET}) \mathrm{X}_{2}+3 \mathrm{ET}^{\circ} \rightarrow 2(\mathrm{ET})_{2} \mathrm{X}
$$

The resulting $(\mathrm{ET})_{2} \mathrm{PF}_{6}$ film was characterized with use of micro-Raman $\left(1468 \mathrm{~cm}^{-1} \mathrm{v}_{3} \mathrm{~A}_{\mathrm{g}}\right)$ and ESR (13 G main component) spectroscopy. This procedure works well with $\mathrm{PF}_{6}^{-}$anion and is expected to be applicable to other electrochemically stable anions such as $\mathrm{ClO}_{4}^{-}, \mathrm{ReO}_{4}^{-}$, $\mathrm{HSO}_{4}^{-}, \quad \mathrm{SF}_{5} \mathrm{CH}_{2} \mathrm{CF}_{2} \mathrm{SO}_{3}^{-}$, etc. This selective electrochemical procedure prepares thin films and is expected to create surface patterns of $(\mathrm{ET})_{2} \mathrm{X}$.

\section{Patterns of neutral ET}

In addition to thin films, we are interested in developing methodologies to prepare ET and (ET) ${ }_{2} \mathrm{X}$ conductive / superconductive surface patterns. Toward that goal, we synthesized a bi-functional ET derivative with dodecanethiol chain that mimics the electronic properties of ET molecule and allows for surface derivatization. The PDMS (polydimethylsiloxane) stamping technique was applied, where the ET derivative, ethylenedithio-methylthio-1,12-dodecanedithio-

tetrathiafulvalene, was stamped onto a gold-on-mica substrate to generate "ET-like" domains. The blank surface was back filled with a hydrophilic reagent, aminoethanethiol hydrochloride (AET). The procedure is outlined in the following scheme.

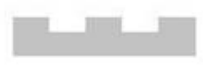

Master

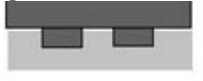

PDMS + Curing agent
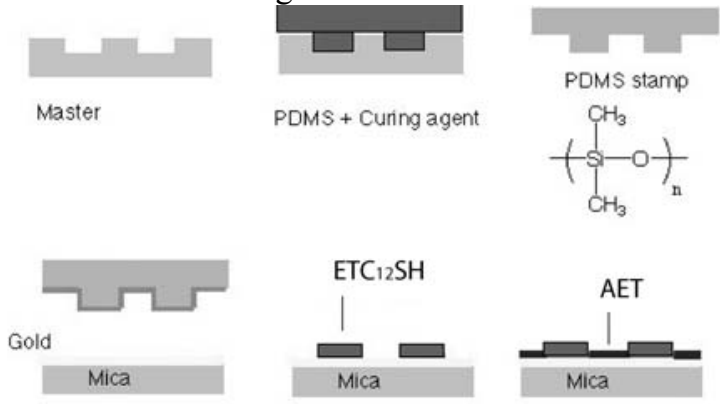

Scheme 1 PDMS stamping technique showing pattern from a master is replicated on a PDMS stamp and imprinted onto a gold/mica substrate with ET derivative ink.
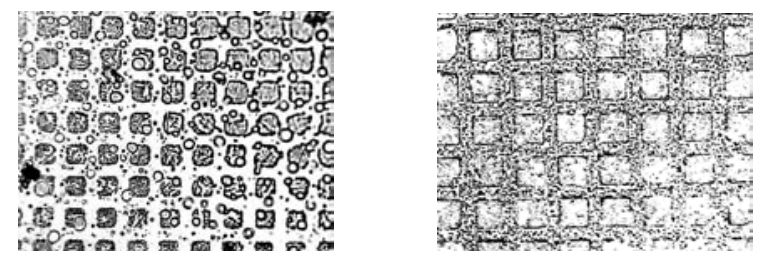

Figure 1a (Left) Neutral ET squares on a gold-on-mica substrate showing $8 \times 8 \mu \mathrm{m}^{2}$ pattern with feature height $\sim 100 \mathrm{~nm}$; $1 \mathrm{~b}$ (Right) Grid pattern ( $7 \mu \mathrm{m}$ wide) of ET salt [likely (ET) ${ }_{3} \mathrm{Cl}_{2} 2 \mathrm{H}_{2} \mathrm{O}$, see text] on a gold-on-mica substrate.
The substrate inked with ET derivative $(1.4 \mathrm{mM})$ and 6 eq. of decanethiol was immersed in a chloroform solution containing ET overnight. The resulting square pattern is shown in Figure 1a. The chemical nature of the squares was verified with use of micro-Raman $(1551 \mathrm{~m}, 1511 \mathrm{w}$, 1494 st) to be identical to that of a pristine ET crystal.

\section{Patterns of ET Salt}

The inverted grid patterns (Figure 1b) are made when the soaking solvent, chloroform, was not freshly distilled and filtered through alumina. The Raman spectrum of the grid indicates two major peaks at $1467\left(\mathrm{C}=\mathrm{C} v_{3} \mathrm{~A}_{\mathrm{g}}\right)$ and $508 \mathrm{~cm}^{-1}\left(\mathrm{C}-\mathrm{S} v_{9} \mathrm{~A}_{\mathrm{g}}\right)$ that are consistent with typical conductive ET salts (Figure 2). One commonly observed side product during electro-crystallization under similar conditions is the ubiquitous $(\mathrm{ET})_{3} \mathrm{Cl}_{2} \cdot 2 \mathrm{H}_{2} \mathrm{O}$. It is likely that this species exists in the presence of small amount of acid and selectively adsorbed onto the hydrophilic AET domains. Detailed characterization is in progress.

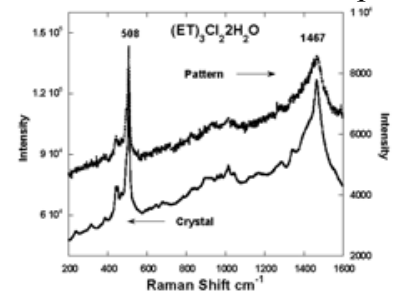

Figure 2 Micro-Raman of the ET salt on the grid pattern derivatized with AET from Figure 1b.

\section{Conclusion}

We have demonstrated new solution techniques to prepare ET salt thin films, both neutral ET and ET salt patterns. Future effort is to optimize the process and bring the resolution into nanometer regions.

\section{References}

[1] T. Ishiguro, K. Yamaji, G. Saito, Organic Superconductors, Vol. 88, 2nd ed., Springer-Verlag, Berlin, Heidelberg, New York, 1998.

[2] J. M. Williams, J. R. Ferraro, R. J. Thorn, K. D. Carlson, U. Geiser, H. H. Wang, A. M. Kini, M.-H. Whangbo, Organic Superconductors (Including Fullerenes): Synthesis, Structure, Properties and Theory, Prentice Hall, New Jersey, 1992.

[3] H. Fujiwara, E. Fujiwara, Y. Nakazawa, B. Z. Narymbetov, K. Kato, H. Kobayashi, A. Kobayashi, M. Tokumoto, P. Cassoux, J. Am. Chem. Soc. 123 (2001) 306.

[4] B. H. Ward, J. A. Schlueter, U. Geiser, H.-H. Wang, E. Morales, J. P. Parakka, S. Y. Thomas, J. M. Williams, P. G. Nixon, R. W. Winter, G. L. Gard, H.-J. Koo, M.-H. Whangbo, Chem. Mater. 12 (2000) 343. 
[5] E. Coronado, J. R. Galán-Mascarós, C. J. Gómez-García, V. Laukhin, Nature (London) 408 (2000) 447.

[6] H. H. Wang, K. L. Stamm, J. P. Parakka, C. Y. Han, Adv. Mater. 2002, In press. 